\title{
Applications of photoemission electron microscopy (PEEM) in magnetism research
}

\author{
Andreas Scholl \\ Ernest Orlando Lawrence Berkeley National Laboratory, 1 Cyclotron Road, Berkeley, CA 94720, USA
}

Received 12 December 2002; accepted 12 December 2002

\begin{abstract}
This paper reviews the application of X-ray photoemission electron microscopy (X-PEEM) to problems in modern magnetism. In particular, the ability to determine the magnetic domain structure of antiferromagnetic thin films will be discussed. Published by Elsevier Science Ltd.
\end{abstract}

\section{Domain imaging with high spatial resolution}

The formation of domains in thin-film magnetic materials is of considerable interest in basic and applied research. In magnetic storage media, such as hard disks, magnetic domains are used for the storage of information at increasingly high density. On the other hand, in magneto-electronic devices magnetic domains are an unwanted product of local variations in the magnetic anisotropy, of the pinning of domain walls, and of the dipolar interaction. Landau and Lifshitz [1] first developed a theory on the origin of magnetic domains, proposing that a process of energy minimization leads to their formation (for a review see also Ref. [2]). Experimental methods have been developed that are capable of imaging the domain configuration over many orders of magnitude in lateral scale. Some methods are based on detecting the magnetic stray field originating from the sample, such as magnetic force microscopy (MFM), Lorentz transmission electron microscopy (TEM) and magnetic indicator techniques. Other techniques directly sense the existence of a magnetization in the sample, which is reflected in a change of the electronic valence band structure of the material. Magnetooptical techniques (MOKE), X-ray magnetic dichroism (XMD), spin-polarized scanning tunneling microscopy (SP-STM) and spin-polarized low energy electron diffraction (SPLEED) belong to this group. X-ray techniques have become more and more popular over the years since they offer high spatial resolution and require relatively

E-mail address: a_scholl@lbl.gov (A. Scholl). little sample preparation, because of the deep penetration of $\mathrm{X}$ rays into matter. Every year, new ultra-bright synchrotron sources, which are needed for high-resolution $\mathrm{X}$-ray microscopy, are going into operation, facilitating easy access of experimenters to X-ray microscopes.

Three types of $\mathrm{X}$-ray microscopes using soft $\mathrm{X}$ rays between 300 and $2000 \mathrm{eV}$ are currently popular: scanning $\mathrm{X}$-ray microscopes, imaging $\mathrm{X}$-ray microscopes and photoemission electron microscopes (PEEM) [3]. The three techniques are distinguished by the method of detecting the $\mathrm{X}$-ray absorption of the sample. Scanning and imaging $\mathrm{X}$-ray microscopes both detect $\mathrm{X}$ rays either transmitted through or reflected by the sample, in contrast to the photoemission electron microscopes, which use electrons photoemitted from the sample. Scanning microscopes use zone-plate optics generating a micro-focused X-ray spot, which is scanned over the sample [4]. Bulk sensitivity can be achieved in a transmission geometry, utilizing the deep penetration of $X$ rays into matter $(\sim 100 \mathrm{~nm})$. Measurements in a reflection geometry are more surface sensitive but suffer from the relatively small reflectivity and a reduced spatial resolution compared to transmission microscopes [5]. One advantage of the reflection geometry is convenience, since samples do not need to be thin. Imaging $\mathrm{X}$-ray microscopes operate in a transmission geometry and also use zone plate optics. A condensor zone-plate illuminates the sample and acts as a simple energy band-pass. A micro zone plate generates a magnified image of the sample transmission. A resolution of 25 $\mathrm{nm}$ has been demonstrated for magnetic domain imaging $[6,7]$. It is noteworthy that transmission microscopes are 
particularly sensitive to the out-of-plane component of the magnetization, whereas reflection microscopes predominantly measure the in-plane magnetization. In-plane sensitivity in the transmission geometry can be achieved by tilting the sample. All pure X-ray techniques are insensitive to the application of external magnetic fields and are therefore particularly suitable for studies of the magnetization reversal process. These microscopes can operate at ambient pressure. Transmission techniques are only moderately sensitive to ultra-thin films because of the relatively weak absorption of $X$ rays in matter. The study of ultra-thin films is the domain of photoemission electron microscopes, which we will describe in detail in the next chapter.

For the study of ferromagnetic domain structures, X-ray microscopes utilize the X-ray magnetic circular dichroism effect, which was discovered by Schütz et al. in 1987 [8]. $\mathrm{X}$-ray magnetic circular dichroism (XMCD) is related to the Faraday and Kerr effect in the visible spectrum but provides orders of magnitude higher contrast because of the large spin-orbit coupling of the involved core levels. The XMCD effect was first utilized in 1993 for the imaging of ferromagnetic domains using a PEEM microscope [9]. The domain contrast of magnetic $3 \mathrm{~d}$ transition metals, e.g. $\mathrm{Fe}, \mathrm{Co}, \mathrm{Ni}$, is a result of the strong dependence of the $L_{2 / 3}$ resonance intensity on the angle between the magnetization of the sample and the circular X-ray polarization. In rare earth ferromagnets, such as $\mathrm{Gd}, \mathrm{Tb}, \mathrm{Sm}$, the $\mathrm{M}_{4,5}$ edges are usually probed. Circularly polarized X rays are available from synchrotron X-ray sources, either by using circularly polarized insertion devices, e.g. helical undulators, or by selecting the radiation of a bending magnet above or below the storage ring plane.

Besides ferromagnets, antiferromagnets are another important class of materials that are used in magneto-electronic devices. Their main application is the pinning of a ferromagnetic layer by an adjacent antiferromagnet, an effect called exchange bias $[10,11]$. The insensitivity of the antiferromagnet to external magnetic fields is utilized to create a magnetic reference. Antiferromagnets do not exhibit magnetic circular dichroism because of their compensated magnetic structure. For the investigation of the domain structure of an antiferromagnet, an effect related to circular dichroism, X-ray magnetic linear dichroism (XMLD), can be used, probing the relative orientation of the antiferromagnetic axis and the linear polarization vector of the incident $X$ rays [12].

In addition to their magnetic sensitivity, X-ray microscopes are sensitive to the elemental and chemical composition of the sample, due to the characteristic position and shape of the near edge X-ray absorption fine structure (NEXAFS) of an element in a particular chemical environment (for a review see Ref. [13]). This sensitivity allows selective probing of individual components in complex systems, e.g. multilayers and alloys, and the correlation of chemical state and magnetic structure.

\section{X-ray photoemission electron microscopy}

$\mathrm{X}$-ray photoemission electron microscopy, abbreviated $\mathrm{X}$-PEEM, is a hybrid technique, combining an X-ray source with a high-resolution electron microscope $[3,14,15]$. This method makes use of the phenomenon that the emission of secondary electrons is proportional to the absorption of $\mathrm{X}$ rays in a near-surface region of the sample. PEEM images these electrons and thus generates a spatial map of the absorption of the sample. Initially by absorbing an X-ray photon a core hole is generated. In low $\mathrm{Z}$ elements this core hole decays predominantly by Auger decay, producing a cascade of secondary electrons. Those electrons whose energy is above the workfunction of the material are able to penetrate the surface and can escape into the vacuum. Electrons that are generated by absorption deep in the bulk of the material have a large chance of being captured. Therefore, PEEM is highly surface sensitive, with a probing depth of only a few nm in metals [16].

Fig. 1 shows a typical synchrotron-PEEM setup, consisting of an X-ray beamline and a secondary-electron microscope $[17,18]$. $X$ rays from the synchrotron are monochromatized using an optical grating monochromator and focused onto the sample. The illuminated area is 30 $\mu \mathrm{m}$ in the case of the presented PEEM-2 microscope, which was developed and is operating at the Advanced Light Source, USA. An aperture in the beamline, selecting $\mathrm{X}$ rays from below, above, or in the storage ring plane determines the polarization of the $\mathrm{X}$ rays. The polarization of the selected $\mathrm{X}$ rays is right circular, left circular, or linear, respectively.

The emitted electrons are accelerated by a strong electric field of typically $15-20 \mathrm{kV}$ between the sample and the microscope. The following four-lens electron optics form a magnified image on an electron-sensitive phosphor. A variable-size aperture in the back-focal plane of the second lens reduces spherical and chromatic image aberrations, significantly improving the spatial resolution of the micro-

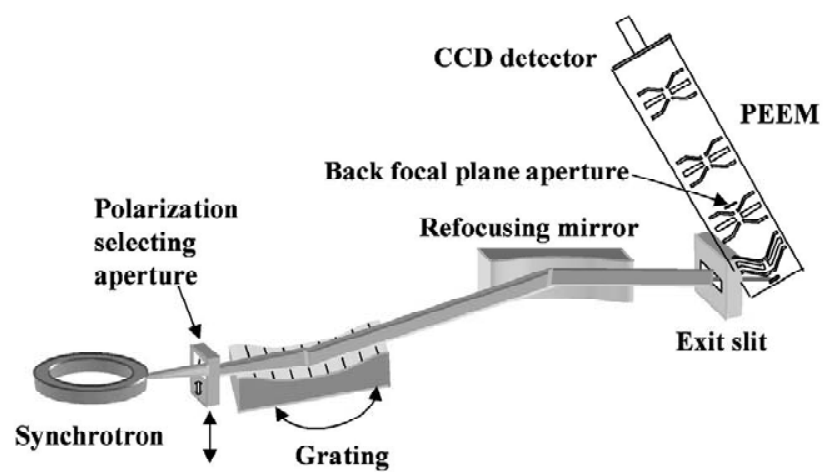

Fig. 1. X-ray photoemission electron microscope and beamline at the Advanced Light Source. Bending magnet radiation is monochromatized and focused onto the sample. The emitted photoelectrons and secondary electrons are imaged with magnification onto a CCD detector by an electron microscope. 

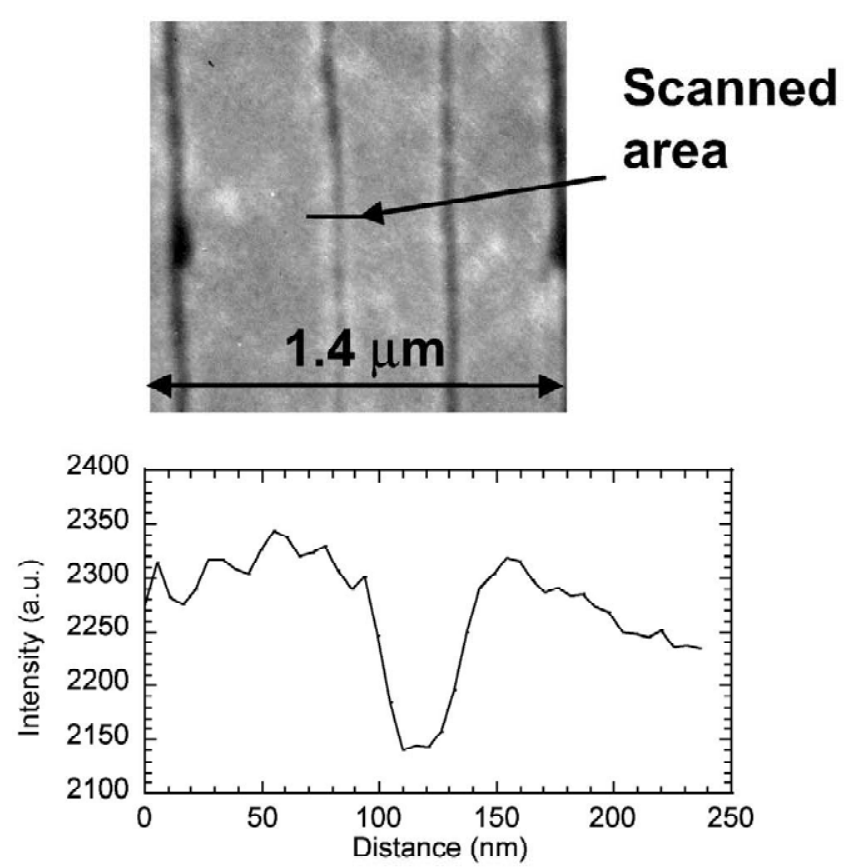

Fig. 2. Electrostatic discharge pattern on $\mathrm{LaFeO}_{3}$. The high-resolution PEEM image and the line scan below show $40 \mathrm{~nm}$ wide structures, demonstrating a spatial resolution of the microscope of clearly below 50 $\mathrm{nm}$. The images were acquired with the photon energy set to the $\mathrm{La} \mathrm{M}_{5}$ edge. The lines in the image are due to a variation in the chemical composition after the discharge.

scope. The aperture limits the angular acceptance of the microscope and at the same time decreases the energy width of the transmitted-electron distribution. Octupol and hexapol elements are used to steer the beam and correct astigmatism. A CCD camera, which is directly coupled to the phosphor by fiber optics, digitizes the image. Some commercial PEEM instead use magnetic lenses and channelplate detectors.

Fig. 2 shows a high-resolution PEEM image, utilizing chemical contrast on $\mathrm{LaFeO}_{3}$ thin film [18]. The grayscale image acquired at the $\mathrm{La} \mathrm{M}_{5}$ edge shows dark lines that are due to a reduced $\mathrm{La}$ concentration in a pattern generated by an electric discharge. The resolved width of the line is about $40 \mathrm{~nm}$, showing that the spatial resolution in this image is clearly less than $50 \mathrm{~nm}$. Using $X$ rays a resolution of PEEM microscopes down to $20 \mathrm{~nm}$ has been reported [18-20]. Using UV excitation an even better resolution of below $10 \mathrm{~nm}$ has been achieved, close to the theoretical limit [21]. In general, the resolution of PEEM microscopes is strongly dependent on the quality of the sample, since the sample is part of the electron optical system. A resolution below $100 \mathrm{~nm}$ has been routinely realized in magnetic imaging [22-27].

\section{Imaging of ferromagnets and antiferromagnets}

As a first example for magnetic microscopy we present images of the ferromagnetic domain structure in a $\mathrm{Pd} / \mathrm{Co} /$ Pd structure (Fig. 3). At the chosen thickness of $0.7 \mathrm{~nm}$, the Co layer, enclosed by two Pd layers, exhibits a perpendicular magnetic anisotropy. Thus, the Co magnetization points either into or out of the sample. The depicted image is the result of dividing two images acquired at the $\mathrm{L}_{3}$ and $\mathrm{L}_{2}$ Co absorption edges using circularly polarized $\mathrm{X}$ rays. Arrows mark the used X-ray energies in the absorption spectrum. Since the X-ray magnetic circular dichroism effect (XMCD) has an opposite sign at the $\mathrm{L}$ edges, dividing the images enhances the magnetic contrast in the ratio image. Non-magnetic contributions, e.g. resulting from variations in the elemental composition or topography, contribute with the same sign
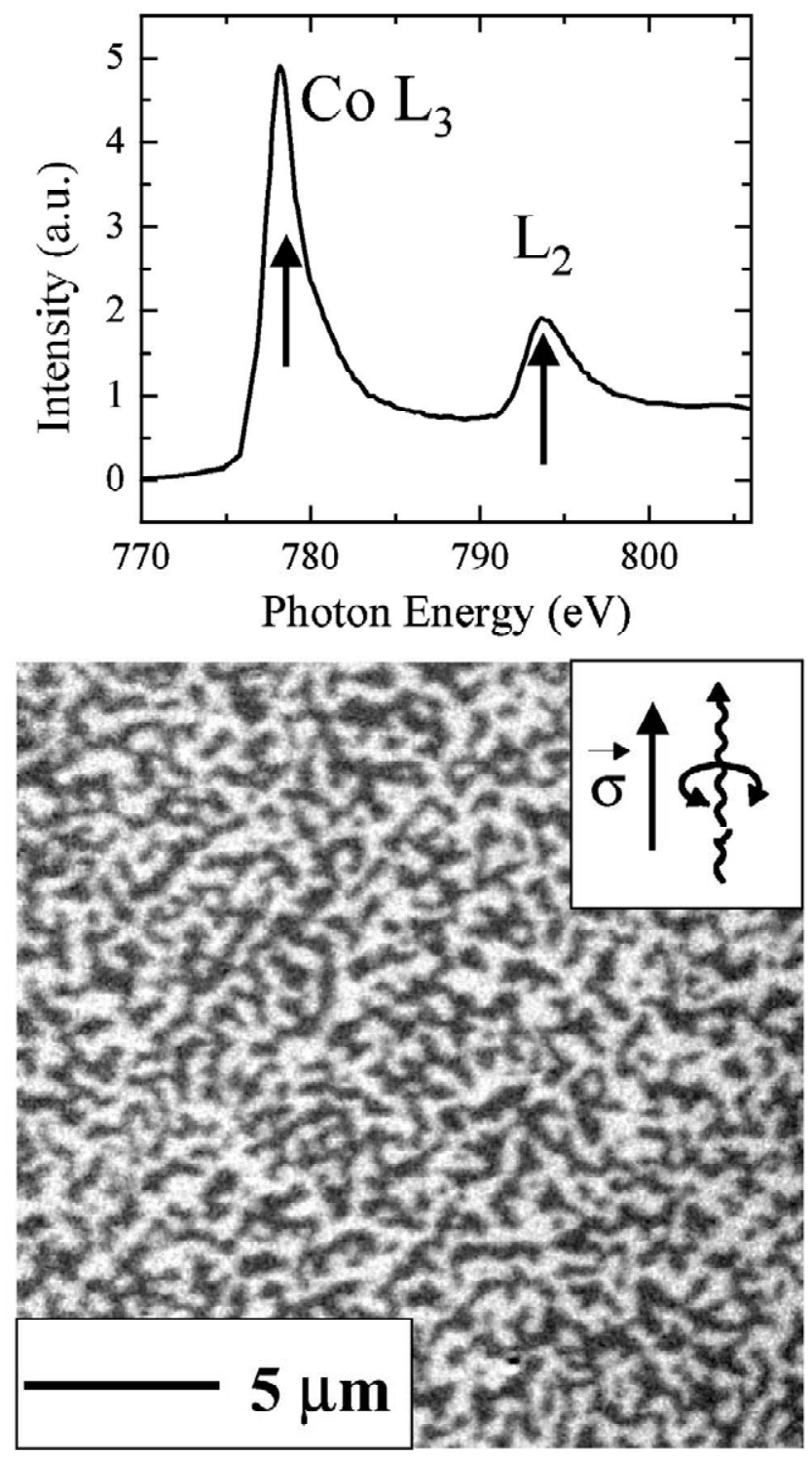

Fig. 3. Ferromagnetic domain pattern of a 0.7 -nm Co layer between two $\mathrm{Pd}$ layers on polycrystalline $\mathrm{Cu}$, imaged using circular polarization. The Co layer exhibits a perpendicular anisotropy and shows worm domains with the magnetization either pointing out of the sample or into the sample. An X-ray absorption spectrum of the Co L edge is shown above. 
and are suppressed. The domain image shows sub-micronsized domains with opposite magnetization, pointing into the plane (bright) and out of the plane (dark). This pattern is typical for an as-grown film with an out-of-plane anisotropy. The dipolar energy is lowered by the formation of worm-like domains, through which the magnetic flux emanating from the sample is closed. The spatial resolution in this image is about $100 \mathrm{~nm}$. The high sensitivity of PEEM is essential for the acquisition of such highresolution images of ultra-thin films.

Fig. 4 illustrates the technique of domain imaging on an antiferromagnetic thin film [22]. The studied 200-nm-thick
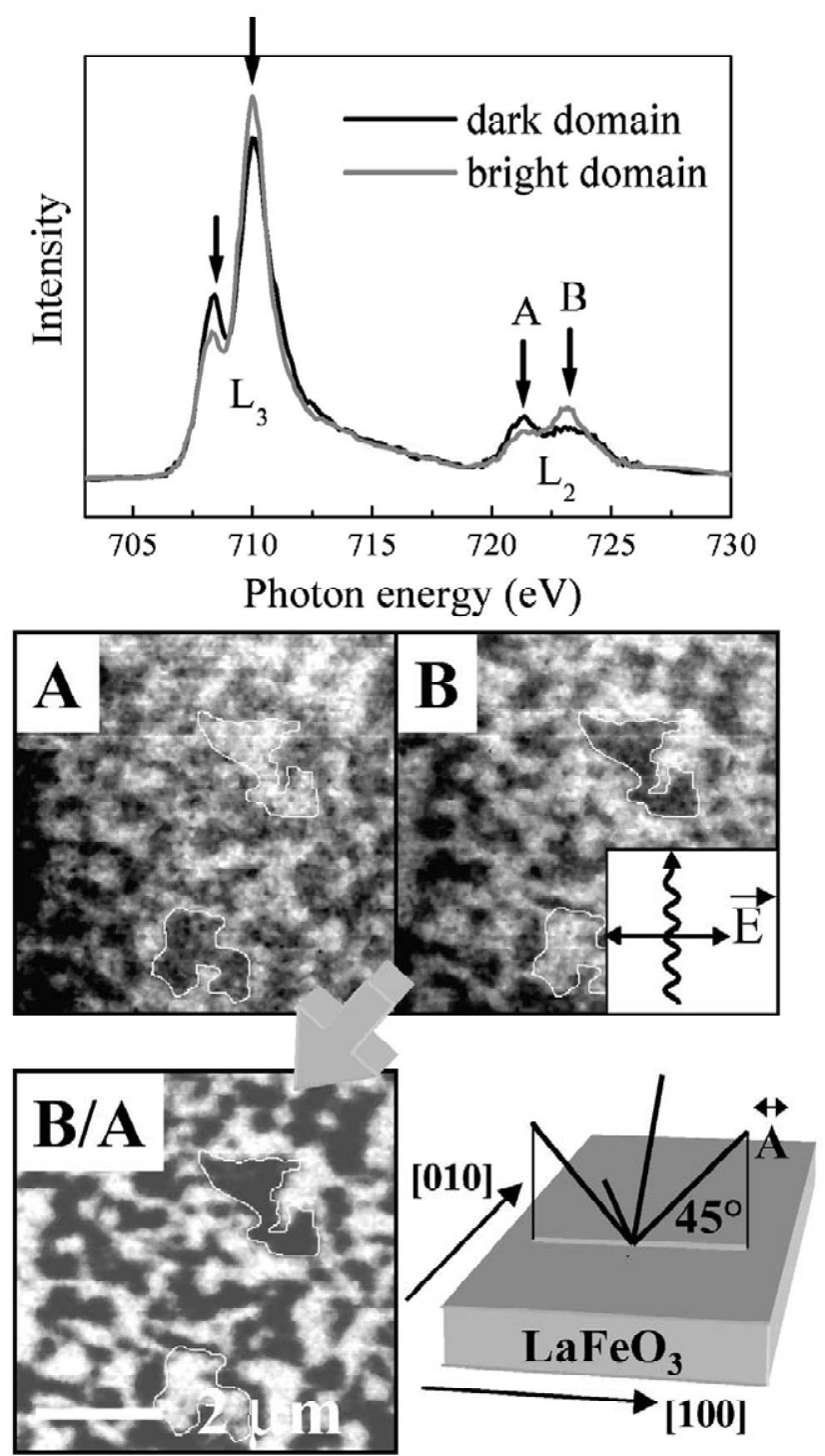

Fig. 4. Magnetic domain structure in a $\mathrm{LaFeO}_{3}$ thin film epitaxially grown on $\mathrm{SrTiO}_{3}(001)$. The images were acquired at the $\mathrm{L}_{2}$ edge using linearly polarized radiation. The color-coded ratio image $\mathrm{B} / \mathrm{A}$ shows the domain structure with enhanced contrast. Local spectra in single antiferromagnetic domains demonstrate the XMLD effect at the L edges. The magnetic structure of the antiferromagnet has been determined from angle- and polarization-dependent images leading to the model, shown at the bottom right.
$\mathrm{LaFeO}_{3}$ film was grown by molecular beam epitaxy on a $\mathrm{SrTiO}_{3}(001)$ substrate. Local spectra of the Fe3p edge are shown on top, acquired in single antiferromagnetic domains using linearly polarized $\mathrm{X}$ rays. The Fe absorption spectra show a magnetic linear dichroism effect at both the $\mathrm{L}_{3}$ and the $\mathrm{L}_{2}$ edge, resulting in a characteristic change in the intensity of the multiplet lines within one absorption resonance. Depending on the relative orientation of the linear X-ray polarization and the spin orientation in the antiferromagnet, domains appear with different brightness in the domain images, which are shown at the bottom of Fig. 4. Images $A$ and $B$ were acquired at the $L_{2}$ edge. Arrows and letters mark the photon energies in the absorption spectrum where the images were acquired. The colorized image at the bottom, the XMLD image, is the result of dividing images $\mathrm{A}$ and $\mathrm{B}$. As already explained above, calculating the ratio image enhances the magnetic contrast. Domains with the in-plane projection of the antiferromagnetic axis parallel to the horizontal X-ray polarization appear brighter than domains with a perpendicular orientation of the antiferromagnetic axis. By changing the azimuthal orientation of the sample and the polarization of the $\mathrm{X}$ rays, the axial orientation of the magnetic moments in the antiferromagnet can be determined. The result of this analysis is shown at the bottom to the right. Four domains have been identified with antiferromagnetic axes along the out-of-plane $\langle 011\rangle$ directions of the cubic $\mathrm{SrTiO}_{3}$ substrate. The domains form groups of two with the same in-plane projection of the axis. These two domains appear with the same brightness in the XMLD ratio image: bright $=$ axis parallel to $X$-ray polarization, dark $=$ axis perpendicular to $\mathrm{X}$-ray polarization.

The magnetic ordering temperature in an antiferromagnet is called the Néel temperature. Above the Néel temperature the magnetic long-range order is destroyed by magnetic fluctuations and the antiferromagnetic domain contrast in images acquired using X-ray dichroism should therefore disappear. The temperature dependence of the magnetic moment can be described to first order by mean field theory. We have verified this hypothesis by acquiring temperature-dependent images on $\mathrm{LaFeO}_{3}$, of which a subset is presented in Fig. 5. Approaching the Néel temperature of the $\mathrm{LaFeO}_{3}$, the domain contrast indeed disappears. It completely recovers after returning to room temperature, confirming the reversibility of the phase transition. The image contrast closely follows the mean field calculated magnetic moment, as demonstrated at the bottom of Fig. 5. The Néel temperature of the thin film, however, appears to be significantly reduced by about $70 \mathrm{~K}$ compared to bulk $\mathrm{LaFeO}_{3}$, which has a Néel temperature of $740 \mathrm{~K}$. This observation has been explained as a strain effect, slightly varying the $\mathrm{Fe}-\mathrm{O}-\mathrm{Fe}$ bonding angle, which is a factor determining the strength of the magnetic superexchange between $\mathrm{Fe}$ atoms.

Taking advantage of the elemental specificity of X-ray PEEM, we have also studied the coupling of the magnetic 

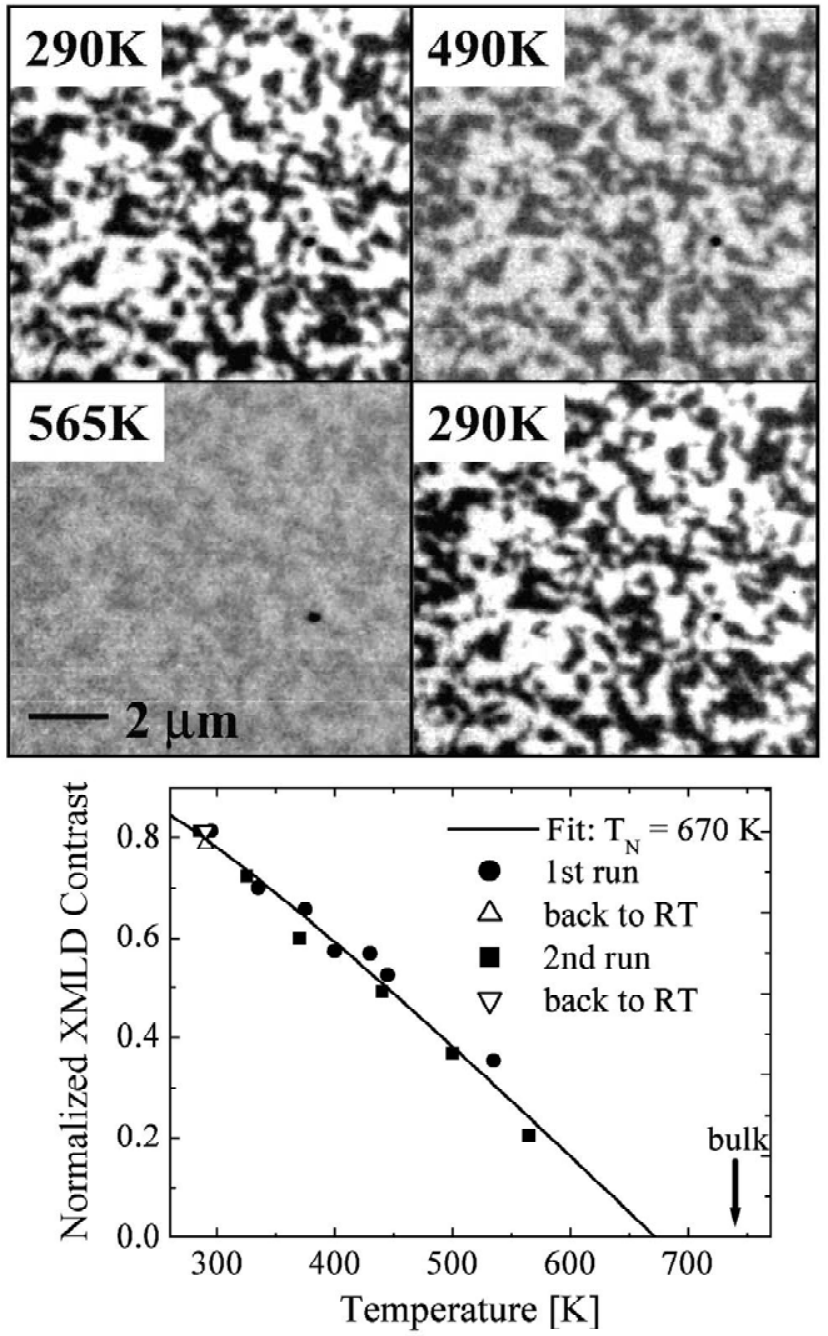

Fig. 5. Temperature dependence of the magnetic image contrast on $\mathrm{LaFeO}_{3}$. The contrast disappears approaching the Néel temperature of the film and reappears at room temperature. The temperature plot indicates a Néel temperature of the thin film below the Néel temperature of the bulk material (marked by arrow).

domain structure between a ferromagnet and an antiferromagnet [23]. In Fig. 6 the domain structure of a Co layer (top row) is compared with the domain structure in an adjacent $\mathrm{LaFeO}_{3}$ layer (bottom row). The 1.2-nm Co/ 200-nm $\mathrm{LaFeO}_{3}$ bi-layer was grown by molecular beam epitaxy on $\mathrm{SrTiO}_{3}$. The XMCD and XMLD effect were used to generate the image contrast on the ferromagnet $\mathrm{Co}$ and the antiferromagnet $\mathrm{LaFeO}_{3}$. The first and second column show images acquired for a different azimuthal rotation of the sample, as explained in the inset. The $90^{\circ}$ rotation of the sample results in a reversal of the contrast in the antiferromagnet (bottom). In the ferromagnet, domains that cannot be distinguished in the first geometry because the magnetization is perpendicular-those regions appear in gray-are distinguished in the $90^{\circ}$-rotated geometry. From the domain images two-dimensional magnetization maps have been generated, shown in the third column. The color code is clarified by the color wheel on the right and the arrows on the left. A parallel coupling of the in-plane components of the magnetic moments in the ferromagnet and the antiferromagnet is apparent. Greenand magenta-colorized domains in the Co layer correspond to green-colorized domains in $\mathrm{LaFeO}_{3}$ (vertical spin axis).

Another example of domain imaging in a coupled ferromagnet-antiferromagnet structure is shown in Fig. 7. $\mathrm{NiO}$ is an antiferromagnet with a Néel temperature of 560 $\mathrm{K}$, well above room temperature. Bi-layer structures consisting of a $\mathrm{NiO}$ layer and a ferromagnetic layer, e.g. Co, exhibit exchange bias. This bias is frozen in the magnetic structure of the system. The microscopic origin of exchange bias is controversial, partly because of the inability of traditional methods to probe the magnetic structure of both the ferromagnet and the antiferromagnet directly at the interface. Its surface sensitivity and sensitivity to ferromagnets and antiferromagnets make PEEM a valuable tool for the investigation of such structures. The system presented in Fig. 7 is $1.5 \mathrm{~nm} \mathrm{Co/NiO}(001)$. The surface of the $\mathrm{NiO}$ bulk single crystal was prepared by ex situ cleaving and annealing in an oxygen atmosphere. The Co layer was grown by e-beam evaporation. The XMLD image, acquired at the Ni2 $\mathrm{p}$ edge (left), and the XMCD image, acquired at the Co $2 p$ edge (right), show the antiferromagnetic domain structure at the surface of the antiferromagnet $\mathrm{NiO}$ and the ferromagnetic domain structure in the Co thin film [26,27]. The brightness of domains in the images corresponds to the direction of the magnetic moments, as explained by the arrows. We observe a parallel coupling of the magnetic moments of the ferromagnet and the interface-near moments of the antiferromagnet, as already discussed in the previous example. Utilizing the elemental and chemical sensitivity of PEEM, we have furthermore investigated the magnetic properties of the $\mathrm{Ni}$ interface layer, which is believed to play an important role in the coupling of the ferromagnet to the antiferromagnet and the formation of exchange bias. The center image shows the ferromagnetic component of the magnetic structure of $\mathrm{NiO}$, revealing a ferromagnetic domain pattern, which closely resembles the domain pattern of the Co layer. This image, which was acquired using circular polarization, demonstrates the existence of uncompensated spins at the surface of the antiferromagnet, linking the magnetic structure of the ferromagnet to the antiferromagnet. Detailed investigations using X-ray spectroscopy and microscopy have shown that the creation of these uncompensated spins is correlated with a chemical reaction at the interface, leading to a reduction of $\mathrm{NiO}$ to $\mathrm{Ni}$ and a partial oxidation of $\mathrm{Co}$ to $\mathrm{CoO}$.

\section{Discussion and conclusion}

Over the last two decades the promise of magnetoelectronic computing and the need for higher-density 


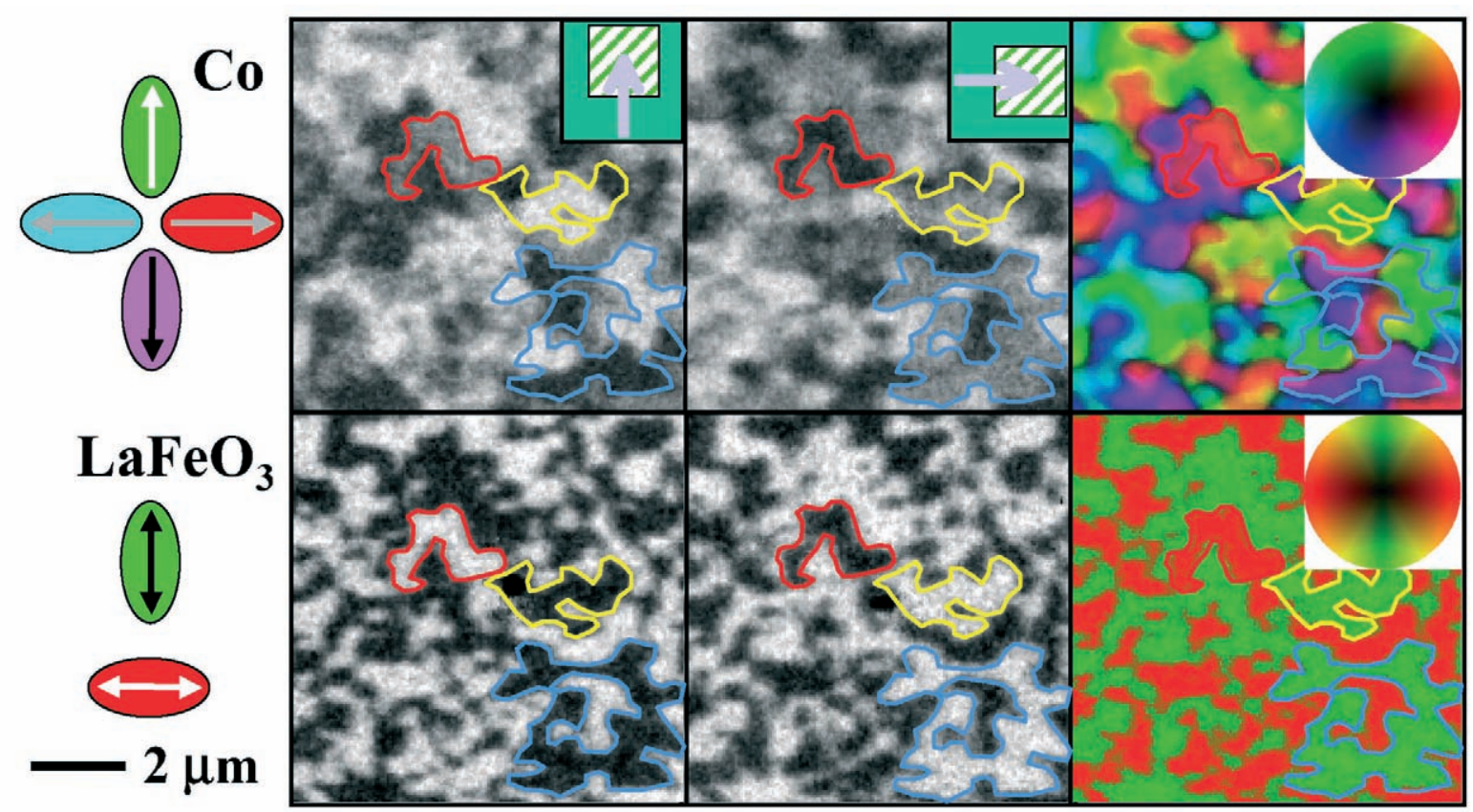

Fig. 6. Two-dimensional magnetization maps in an exchange coupled $\mathrm{Co} / \mathrm{LaFeO}_{3}$ bi-layer. Magnetic domain images were acquired in two geometries on $\mathrm{Co}$ using magnetic circular dichroism and on $\mathrm{LaFeO}_{3}$ using magnetic linear dichroism. The color-coded maps were generated from the domain images. The images demonstrate parallel coupling between the layers.

magnetic storage devices has led to the development of nano-scale magnetic structures. The ongoing reduction in lateral scale and in thickness of the magnetic layers illustrates the need for high-sensitivity magnetic detection techniques, ideally with microscopy capability. The classic tools of magnetism research, neutron diffraction and magnetometry, have been vital for the development and understanding of novel magnetic materials and still play a major role in this area. Their limitation in regard to spatial resolution and thin film sensitivity led to the development of new techniques, in particular the magneto-optical Kerr

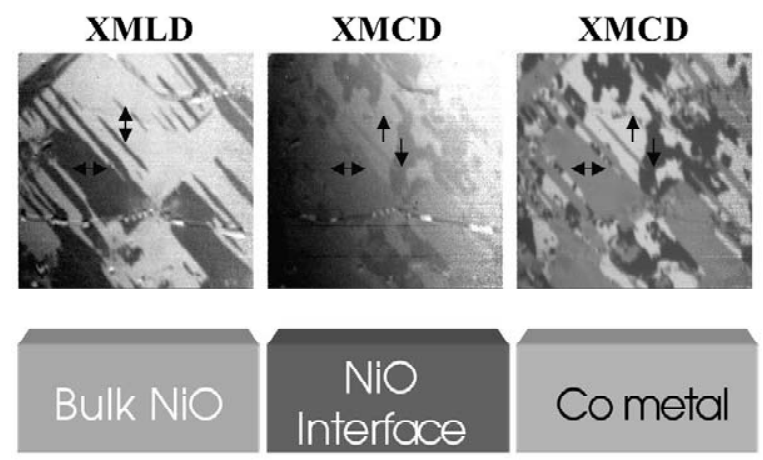

Fig. 7. Antiferromagnetic domains (left) and ferromagnetic domains (right) in $\mathrm{Co} / \mathrm{NiO}(001)$. The $\mathrm{Ni}$ interface magnetic structure is shown in the center. Ferromagnetic Ni spins $(\sim 1$ monolayer thickness $)$ at the interface between $\mathrm{NiO}$ and $\mathrm{Co}$ show magnetic circular dichroism linking the ferromagnetically ordered Co layer to the antiferromagnet $\mathrm{NiO}$. The ferromagnetic domain structure of the interface layer is identical to the domain structure of Co. The images also demonstrate the high sensitivity of X-PEEM to small concentrations of magnetic moments. effect and magnetic force microscopy (MFM), which today are standard tools in laboratories. Kerr microscopes, which operate using visible light, are limited however by the diffraction and can only reach a spatial resolution on the order of several $100 \mathrm{~nm}$. MFMs, which are based on the atomic force microscopy technique, have higher resolution down to tens of nanometers or better. However, MFM does not directly probe the magnetization of the sample; instead it detects its stray field using a magnetic tip. The tip magnetization can furthermore influence the sample magnetization and obscure the domain structure in soft magnetic materials. X-ray microscopes using the linear and circular dichroism effect allow quantitative measurements of the atomic magnetic moments at very high spatial resolution, comparable with the best magnetic force microscopes and distinctly better than microscopes utilizing optical MOKE. Thus they have become a very important and versatile tool for the investigation of magnetic domains and magnetic coupling in multi-layer thinfilm structures. In addition, synchrotron-based X-ray techniques have the unique capability of selectively probing one component of a complex sample, by tuning the X-ray energy to the characteristic absorption edge or NEXAFS resonance of the material.

In multi-layer structures the interface region between layers plays an important role, often dominating the magnetic properties of a material. The interface properties can lead to changes in the magnetic anisotropy, as we have demonstrated in the case of thin Co films, which depending on the environment and thickness exhibit an in-plane or out-of-plane anisotropy. The interface is also important 
for exchange bias and for spin transport phenomena. Surface sensitivity is essential to experimentally discriminate the interface signal from the signal originating from the bulk. In combination with the elemental and chemical selectivity of $\mathrm{X}$ rays, $\mathrm{X}$-ray PEEM in particular is an exceptionally powerful tool for the investigation of interface and surface properties.

The shortest length scale over which the magnetization in a material typically varies is the width of a domain wall $\sqrt{A / K_{x}}$, where $A$ is the exchange constant and $K_{x}$ is either the anisotropy or the stray field energy constant. $\sqrt{A / K_{x}}$ is also called exchange length. This length lies typically between $1 \mathrm{~nm}$ and several $100 \mathrm{~nm}$, depending on the material and shape of the magnetic sample. Magnetic microscopy techniques should therefore ideally reach a resolution equal or better than the exchange length. The typical size of magnetically isolated particles forming single domains, e.g. in patterned or polycrystalline materials, falls within this range. Currently, X-ray PEEM and most other competing imaging techniques only fulfill this criterion for relatively soft materials with a rather large exchange length. Therefore, progress in regard to the achievable spatial resolution is required. Aberration correction of a conventional PEEM is a possible solution in order to achieve a spatial resolution of well below $10 \mathrm{~nm}$. Current development projects are exploring the possibility of aberration correction in the USA [28] and in Germany. We expect that the next-generation microscope will offer superior spatial resolution, which will be sufficient to resolve the finest details of the domain structure in magnetic materials.

Another promising new development is the application of PEEM to the investigation of time-resolved magnetic processes. Synchrotron sources produce X-ray pulses with a pulse length of, in the case of the Advanced Light Source, 50-100 ps at repetition rate of between 3 and 500 $\mathrm{MHz}$, depending on the mode of operation. Magnetization processes such as precession and damping typically occur on time scales of $10 \mathrm{ps}$ to $1 \mathrm{~ns}$. Therefore time-resolved $\mathrm{X}$-ray microscopy covers a large part of the interesting time range, with the particular advantages of high spatial resolution and elemental specificity as explained above.

In conclusion, X-ray PEEM, due to its high sensitivity, high spatial resolution, and elemental specificity, is a very versatile tool for the investigation of the ever more complex magnetic structures and devices used in modern magnetism. In the future, aberration-corrected microscopes will allow us to study magnetic structures and magnetization dynamics in even greater detail than is possible today.

\section{Acknowledgements}

The author wants to thank S.-B. Choe, F. Nolting, H. Ohldag, and J. Stöhr, who contributed to this work. This work was supported by the Director, US Department of Energy, Office of Basic Energy Sciences, under Contract No. DE-AC03-76SF00098.

\section{References}

[1] Landau LD, Lifshitz E. On the theory on the dispersion of magnetic permeability in ferromagnetic bodies. Phys $\mathrm{Z}$ Sowjetunion 1935;8:153-69.

[2] Hubert A, Schäfer R. Magnetic domains: the analysis of magnetic microstructures. Berlin: Springer, 1998.

[3] Stohr J, Padmore HA, Anders S, Stammler T, Scheinfein MR. Principles of X-ray magnetic dichroism spectromicroscopy. Surface Review \& Letters 1998;5:1297-308.

[4] Warwick $T$ et al. A new bend-magnet beamline for scanning transmission X-ray microscopy at the Advanced Light Source. J Synchrotron Radiat 2002;9(4):254-7.

[5] Friedrich J et al. Scanning microscopy of magnetic domains using the Fe 3p core level transverse magneto-optical Kerr effect. Journal of Applied Physics 1999;85:4610-2.

[6] Denbeaux G et al. A full field transmission X-ray microscope as a tool for high-resolution magnetic imaging. IEEE Transactions on Magnetics 2001;37:2764-6.

[7] Fischer $P$ et al. Element-specific imaging of magnetic domains at 25 $\mathrm{nm}$ spatial resolution using soft X-ray microscopy. Rev Sci Instrum 2001;72:2322-4.

[8] Schutz G et al. Absorption of circularly polarized X-rays in iron. Phys Rev Lett 1987;58:737-40.

[9] Stohr J et al. Element-specific magnetic microscopy with circularly polarized X-rays. Science 1993;259:658-61.

[10] Nogues J, Schuller IK. Exchange bias. J Magn Magn Mater 1999;192:203-32.

[11] Berkowitz AE, Takano K. Exchange anisotropy. J Magn Magn Mater 1999;200:552-70.

[12] van der Laan $G$ et al. Experimental proof of magnetic X-ray dichroism. Phys Rev B 1986;34:6529-31.

[13] Stöhr J. NEXAFS spectroscopy. Berlin: Springer, 1992.

[14] Hillebrecht FU. Magnetic imaging. J Phys Condens Matter 2001;13:11163-80.

[15] Tonner BP et al. The development of electron spectromicroscopy. J Electron Spectrosc Relat Phenom 1995;75:309-32.

[16] Nakajima R, Stohr J, Idzerda YU. Electron-yield saturation effects in L-edge X-ray magnetic circular dichroism spectra of $\mathrm{Fe}$, $\mathrm{Co}$, and Ni. Phys Rev B 1999;59:6421-9.

[17] Scholl A, Ohldag H, Nolting F, Stohr J, Padmore HA. X-ray photoemission electron microscopy, a tool for the investigation of complex magnetic structures (invited). Rev Sci Instrum 2002;73:1362-6.

[18] Anders S et al. Photoemission electron microscope for the study of magnetic materials. Rev Sci Instrum 1999;70:3973-81.

[19] Bauer E. In: Rosei R, editor, Chemical, Structural, and Electronic Analysis of Heterogeneous Surfaces on Nanometer Scale, Dordrecht: Kluwer Acad. Publ., 1997.

[20] De Stasio G et al. MEPHISTO spectromicroscope reaches $20 \mathrm{~nm}$ lateral resolution. Rev Sci Instrum 1999;70:1740-2.

[21] Rempfer GF, Griffith OH. The resolution of photoelectron microscopes with UV, X-ray and synchrotron excitation sources. Ultramicroscopy 1989;27:273-300.

[22] Scholl A et al. Observation of antiferromagnetic domains in epitaxial thin films. Science 2000;287:1014-6.

[23] Nolting F et al. Direct observation of the alignment of ferromagnetic spins by antiferromagnetic spins. Nature 2000;405:767-9.

[24] Stohr $\mathbf{J}$ et al. Images of the antiferromagnetic structure of a 
$\mathrm{NiO}(100)$ surface by means of X-ray magnetic linear dichroism spectromicroscopy. Phys Rev Lett 1999;83:1862-5.

[25] Thomas L et al. Oscillatory decay of magnetization induced by domain-wall stray fields. Phys Rev Lett 2000;84:3462-5.

[26] Ohldag $\mathrm{H}$ et al. Spectroscopic identification and direct imaging of interfacial magnetic spins. Phys Rev Lett 2001;87:247201/1-4.
[27] Ohldag $\mathrm{H}$ et al. Spin reorientation at the antiferromagnetic $\mathrm{NiO}(001)$ surface in response to an adjacent ferromagnet. Phys Rev Lett 2001;86:2878-81.

[28] Feng $\mathbf{J}$ et al. Modeling the acceleration field and objective lens for an aberration corrected photoemission electron microscope. Rev Sci Instrum 2002;73:1514-7. 